\section{Thrombolytic therapy for submassive pulmonary embolus? PRO viewpoint}

\author{
Luke S Howard
}

If we had robust evidence one way or the other to inform us on the use of thrombolytics in submassive pulmonary embolus (PE), we would not need this debate. But, we do not and, so, we do. The stakes are considered high both in favour and against thromobolysing submassive PE, so we cannot brush the debate under the carpet while we await the evidence. It is accepted that high-risk/massive PE, defined as haemodynamic instability, merits aggressive treatment due to unacceptable mortality, which outweighs the risk of haemorrhage. At the other end of the spectrum, patients with low-risk PE do not, such that they may even be treated as outpatients.

This leaves a grey area in between. When faced with a patient with a large thrombus load with a right ventricle (RV) that is dilated and pressure-loaded, but who is normotensive, as the attending clinician, we know they are at increased risk of death and long-term complications, such as chronic thromboembolic pulmonary hypertension (CTEPH). ${ }^{1}{ }^{2}$ We may fall back on the Hippocratic Oath to 'first, do no harm', but the fuller translation reads 'I will use treatments for the benefit of the ill in accordance with my ability and my judgment, but from what is to their harm and injustice I will keep them'. Thus, rather than hide behind a lack of evidence, we must review what we have and come to a balanced decision, and justify it.

In proposing the argument that submassive PE should be treated with thrombolysis, we must first accept that direct mortality due to the PE itself, not confounding conditions, remains unacceptably high with anticoagulation alone. A more aggressive strategy is required. As long as the benefits of thrombolysis outweigh the risks, then thrombolysis offers the best currently available approach. When this is coupled with the further benefits of likely reduction in CTEPH, the case becomes even stronger.

Correspondence to Dr Luke S Howard, National Pulmonary Hypertension Service, Hammersmith Hospital, Imperial College Healthcare NHS Trust, Du Cane Road, London W12 OHS, UK; l.howard@imperial.ac.uk
The American Heart Association $(\mathrm{AHA})^{3}$ has proposed a definition of submassive PE as either:

- RV dysfunction, defined as RV dilation on echocardiography or CT, systolic dysfunction on echocardiography, elevation of brain natiuretic peptide (BNP)/N-terminal pro BNP (NTproBNP) or evidence of new RV strain on ECG; or

- myocardial necrosis, defined as elevation of troponin I or T.

These measures either alone or in combination have been shown in many studies to be associated with worse survival, but this definition itself has not been prospectively studied. Jimenez et $a l^{4}$ collected data from 591 normotensive PE patients (those who had received thrombolysis at the physician's discretion were not included) and showed that there was a $10 \%$ overall mortality. Pulmonary embolism may often occur on the background of other serious medical conditions, which may themselves lead to death. In these cases, it is hard to see how thrombolysis could improve the outcome, but Jimenez et al demonstrated that the rate of PE death, as opposed to all cause death, was $6.7 \%$. When some of the criteria for submassive PE were applied, the rate of PE-related mortality increased to $11 \%$ with a positive troponin, $12 \%$ with echocardiographic evidence of RV dysfunction and $10 \%$ with complete compression ultrasound evidence of deep vein

Presented with data such as these, we may ask why studies of thrombolysis have not shown improvement in mortality. The reasons may be twofold. The first is that patients recruited into studies may have less severe disease, either due to a looser definition of submassive PE or due to those with more severe signs of right heart dysfunction not being recruited at the physician's discretion in order they be thrombolysed outside the protocol. Looking at the largest trial to date of thrombolysis in submassive $\mathrm{PE}^{5}$ only $31 \%$ of patients in the standard treatment arm had RV dysfunction on echocardiography and the mortality rate in that same arm was just over $2 \%$. Contrast this with $>10 \%$ mortality in the study by Jimenez et $a l^{4}$ if patients had one or more of RV thrombosis (DVT). dysfunction, positive troponin or DVT. The AHA document ${ }^{3}$ pools all the randomised thrombolysis studies in PE up to 2011, and only one has more than 100 patients in each $\mathrm{arm}^{5}$ and it is therefore also not surprising to appreciate that these studies will be underpowered to detect a significant reduction in mortality. The studies were performed in different categories of patients and over 40 years, making pooling difficult, although it is worth noting there was a $30 \%$ nonsignificant reduction in mortality in the patients receiving thrombolysis. We can perhaps therefore conclude that trials may be under-representative of the true mortality of submassive PE. Of interest, however, a study not included, due to later publication, ${ }^{6}$ randomised only patients with documented RV dysfunction on echocardiography, thus enriching the population, and in this study there was a significant reduction in PE-related mortality with 6/35 patients dying in the heparin group and $0 / 37$ dying in the thrombolysis group.

The second reason that trials so far may not have shown a reduction in mortality is that they allow for thrombolysis in the event of haemodynamic collapse. To capture this effect, studies often combine mortality with haemodynamic collapse to detect a treatment response. ${ }^{5} 7$

The authors of the AHA document suggest that since the mortality seen in trials for submassive PE is less than 3\%, even with highly effective therapy reducing mortality by 30\%, trials will be unsuccessful in proving a mortality benefit, and thus surrogate endpoints should be used. ${ }^{3}$ Since we know that PE-related mortality is linked to RV dysfunction which in turn is largely caused by clot burden ${ }^{2}$ (likely to be a combination of burden in the lung and leg veins, since DVT increases the risk of death ${ }^{4}$ ), two good possible surrogates for mortality are haemodynamics and clot resolution.

A recent randomised study of thrombolysis in 'moderate' pulmonary embolism measured systolic pulmonary artery pressure (sPAP) as estimated on echocardiography as its primary endpoint. Thrombolysis versus anticoagulation resulted in a significantly lower sPAP at $48 \mathrm{~h}$. Showing this fall in sPAP at such an early time point is critical to its credibility as a surrogate for PE-related mortality. This has also been documented in several other studies. ${ }^{3}$ If only patients with RV dysfunction are considered, thrombolysis improves early RV function and BNP compared with placebo. ${ }^{6}$

The rate of clot resolution over the first $24 \mathrm{~h}$ has been shown to change very little with heparin, whereas with thrombolysis, 
there is a $30 \%-35 \%$ reduction in perfusion defect. ${ }^{3}$ For patients with RV dysfunction, it is this early resolution of clot with thrombolysis which will prove to be beneficial in reducing early complications. One may also postulate that earlier resolution of DVT with thrombolysis may also improve outcomes, since further embolisation may be fatal. Of note, the study by Fasullo et $a l^{6}$ showed a trend towards better DVT resolution in patients receiving thrombolysis, only just missing statistical significance. It will take a large and wellconstructed trial with mortality comparable with real-life data in the placebo arm to show mortality benefit with thrombolysis; until that time, surrogate data strongly suggest thrombolysis directly reduces the risk factors for death.

In addition to early PE-related mortality, there are important long-term outcomes from PE, in particular CTEPH. While it may not be common in all comers with PE, $2 \%-4 \%$ of cases, ${ }^{1}$ it is likely to be much more common in those patients who have had submassive PE. No study has been performed rigorously using right heart catheterisation to study the incidence of CTEPH postsubmassive $\mathrm{PE}$, especially given the variation in definition. Nonetheless, despite its likely low incidence, in itself it carries a significant risk of death. ${ }^{1}$ Even if amenable to surgical endarterectomy, this is a very major surgical operation with its own mortality $(<5 \%)$ and morbidity. ${ }^{8}$ The argument may be less persuasive than early mortality since the associations are less direct, but adds to the weight of the argument in favour of thrombolysis.

Many studies referenced in the AHA statement, ${ }^{3}$ in particular those by Kline et $a l^{9}$ and Fasullo et $a l^{6}{ }^{6}$ show lower longterm pulmonary arterial pressures and better RV function in those who were treated upfront with thrombolysis. How much of the improvement in RV function relates to more rapid relief of RV afterload cannot be certain, but the lower pulmonary pressures would suggest better clot resolution, which in itself is associated with lower 6-month mortality. ${ }^{10}$ Why anticoagulation fails to catch up with thrombolysis is not fully understood, but studies of plasmin-mediated cleavage of fibrin have demonstrated resistance to fibrinolysis in patients with CTEPH compared with those with acute PE. ${ }^{11}$ Allowing endogenous clot lysis to take place 'passively' using heparin alone may not be sufficient therefore to prevent progression to CTEPH in some patients. Identifying these patients upfront is not currently possible, but those with acute pulmonary hypertension are at the greatest risk of CTEPH. ${ }^{1}$ That many patients presenting with CTEPH have in the past received thrombolysis ${ }^{12}$ is not relevant, since many of these patients will have suffered previously unresolved PE.

While there can be little doubt that thrombolysis leads to faster and possibly more complete clot resolution, it is the risk of serious bleeding, in particular intracranial haemorrhage, which causes reticence when considering thrombolysis. This is a real concern and should not be taken lightly, but what we must consider is the balance of benefit against risk. A recent very large registry study of nearly 16000 patients with PE showed a non-significant trend towards increased bleeding-related mortality at 90 days in those who received thrombolytics. ${ }^{13}$ While the mortality was nearly double in the thrombolysis group $(1.16 \%$ vs $0.61 \%$, $\mathrm{p}=0.16$ ), in absolute terms the rate was very low. Given that the entire cardiac output transits through the lung, it has been argued that lower doses of thrombolytics could be used with equal efficacy. Two recent studies have done just this, ${ }^{14} 15$ showing equivalent clot resolution and lower rates of bleeding, although the numbers remain small in comparison with large registry studies.

It is the intention of the PEITHO investigators that this debate is settled once and for all, ${ }^{7}$ but for the reasons explained above, namely, lower overall than expected mortality and the use of rescue thrombolysis, the PE-related mortality signal may be watered down. Until the data provide a clear answer, we must use our judgement.

In summary, outcomes in patients with true submassive $\mathrm{PE}$ remain unacceptably high and thrombolysis has been shown to improve surrogate outcomes for mortality as well as long-term complications. The risks from thrombolysis are low, and when reduced doses are used, evidence so far suggests no decrease in benefit, but a further reduction in bleeding. The next patient you see with submassive PE will want you to act on your best judgement rather than hide behind a 'lack of evidence'.

Disclaimer The views expressed in this article do not necessarily represent the personal views or practice of the author, but have been written to stimulate debate.

Competing interests Luke Howard has received research funding, speaker fees and fees for consultancy from Bayer.

Provenance and peer review Commissioned; internally peer reviewed.
To cite Howard LS. Thorax 2014:69:103-105.

Published Online First 26 April 2013

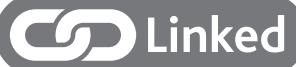

- http://dx.doi.org/10.1136/thoraxinl-2012-202900

- http://dx.doi.org/10.1136/thoraxjnl-2013-203746

http://dx.doi.org/10.1136/thoraxjnl-2013-204193

Thorax 2014;69:103-105.

doi:10.1136/thoraxjnl-2013-203413

\section{REFERENCES}

1 Piazza G, Goldhaber SZ. Chronic thromboembolic pulmonary hypertension. $N$ Engl J Med 2011:364:351-60.

2 Furlan A, Aghayev A, Chang CC, et al. Short-term mortality in acute pulmonary embolism: clot burden and signs of right heart dysfunction at CT pulmonary angiography. Radiology 2012;265:283-93.

3 Jaff MR, McMurtry MS, Archer SL, et al. Management of massive and submassive pulmonary embolism, iliofemoral deep vein thrombosis, and chronic thromboembolic pulmonary hypertension: a scientific statement from the American Heart Association. Circulation 2011;123:1788-830.

4 Jimenez D, Aujesky D, Moores L, et al. Combinations of prognostic tools for identification of high-risk normotensive patients with acute symptomatic pulmonary embolism. Thorax 2011;66:75-81.

5 Konstantinides S, Geibel A, Heusel G, et al. Heparin plus alteplase compared with heparin alone in patients with submassive pulmonary embolism. N Engl J Med 2002:347:1143-50.

6 Fasullo S, Scalzo S, Maringhini G, et al. Six-month echocardiographic study in patients with submassive pulmonary embolism and right ventricle dysfunction: comparison of thrombolysis with heparin. Am J Med Sci 2011;341:33-9.

7 PEITHO Investigators. Single-bolus tenecteplase plus heparin compared with heparin alone for normotensive patients with acute pulmonary embolism who have evidence of right ventricular dysfunction and myocardial injury: rationale and design of the Pulmonary Embolism Thrombolysis (PEITHO) trial. Am Heart J 2012;163:33-8.

8 Pepke-Zaba J, Delcroix M, Lang I, et al. Chronic thromboembolic pulmonary hypertension (CTEPH): results from an international prospective registry. Circulation 2011;124:1973-81.

9 Kline JA, Steuerwald MT, Marchick MR, et al. Prospective evaluation of right ventricular function and functional status 6 months after acute submassive pulmonary embolism: frequency of persistent or subsequent elevation in estimated pulmonary artery pressure. Chest 2009;136: 1202-10.

10 Meneveau N, Ider O, Seronde MF, et al. Long-term prognostic value of residual pulmonary vascular obstruction at discharge in patients with intermediate- to high-risk pulmonary embolism. Eur Heart J 2013;34:693-701.

11 Miniati M, Fiorillo C, Becatti M, et al. Fibrin resistance to lysis in patients with pulmonary hypertension other than thromboembolic. Am J Respir Crit Care Med 2010;181:992-6.

12 Pengo $\mathrm{V}$, Lensing $\mathrm{AW}$, Prins $\mathrm{MH}$, et al. Incidence of chronic thromboembolic pulmonary hypertension after pulmonary embolism. $N$ Engl I Med 2004;350:2257-64.

13 Riera-Mestre A, Jimenez D, Muriel A, et al. Thrombolytic therapy and outcome of patients with 
an acute symptomatic pulmonary embolism. J Thromb Haemost 2012;10:751-9.

14 Wang C, Zhai Z, Yang Y, et al. Efficacy and safety of low dose recombinant tissue-type plasminogen activator for the treatment of acute pulmonary thromboembolism: a randomized, multicenter, controlled trial. Chest 2010;137:254-62.
15 Sharifi M, Bay C, Skrocki L, et al. Moderate pulmonary embolism treated with thrombolysis (from the "MOPETT" Trial). Am J Cardiol 2013;111(2): 273-7. 